\title{
XXIV. On the resistance of telegraphic electromagnets
}

\section{Oliver Heaviside}

To cite this article: Oliver Heaviside (1878) XXIV. On the resistance of telegraphic electromagnets, Philosophical Magazine Series 5, 6:36, 177-185, DOI:

$10.1080 / 14786447808639495$

To link to this article: http://dx.doi.org/10.1080/14786447808639495

曲 Published online: 13 May 2009.

Submit your article to this journal $[\pi$

Џ Article views: 5

Q View related articles $\asymp$ 


\section{$\left[\begin{array}{ll}177 & ]\end{array}\right.$ \\ XXIV. On the Resistance of Telegraphic Electromagnets. By Oliver Heaviside*.}

1. THERE appears to be some uncertainty regarding the 1 proper resistance which electromagnets for signallingpurposes should have-whether a receiving instrument should have a resistance equal to that of the remainder of the circuit, or a half, or a fifth, or some other fixed fraction thereof. Practical experience, especially with high-speed instruments, has shown that the resistances in general use are too high-and that advantage is gained by reducing the resistance of an electromagnetic receiving instrument, employing fewer windings of a thicker wire in place of more windings of a thinner, thereby reducing the self-induction as well. Ohm, as long ago as 1826, showed that the resistance of a galvanometer should equal that of the rest of the circuit in which it is placed, to obtain the maximum magnetic force. When the correction needed on account of the thickness of the insulating covering of the wire is also reckoned, then the thickness of the wire of the galvanometer should be such that the external resistance is to the resistance of the galvanometer-coil as the diameter of the covered wire is to the diameter of the wire itself (Maxwell, vol. ii. p. 321).

Now if, in telegraphic signalling, sufficient time were allowed during every signal (positive, negative, or no current) for the full effect to be produced in the circuit by the electromotive force, or for the current to entirely die away, the above result would hold good also. But such is not the case ; for by reason of electrostatic and electromagnetic induction, the current has not time to reach its full strength during every signal. On a land-line, unless it is very long, electromagnetic induction is the principal retarding cause; and it is this case which is here considered.

2. Let there be a simple harmonic variation of electromotive force

\section{E sin $m t$,}

where $\mathrm{E}$ and $m$ are constants, and $t$ is the time, in a circuit of resistance $R$ and electromagnetic capacity L. The equation of the current is

$$
\mathrm{E} \sin m t=\left(\mathbf{R}+\mathrm{L} \frac{d}{d t}\right) \gamma, \ldots . . .
$$

where $y$ is the current at time $t$. The solution of (1) is

$$
\gamma=\frac{\mathrm{E}}{\sqrt{\mathrm{R}^{2}+\mathrm{L}^{2} m^{2}}} \sin \left(m t-\tan ^{-1} \frac{m \mathrm{~L}}{\mathrm{R}}\right),
$$

Plit. Mag. S. 5. Vol. 6. No. 36. Sept. 1878. 
neglecting a vanishing term. The amplitude of the currentwaves is thus reduced from

$$
\frac{\mathbf{E}}{\overline{\mathbf{R}}}
$$

(what it would be were there no retardation) to

$$
\Gamma=\frac{\mathbf{E}}{\sqrt{\mathrm{R}^{2}+\mathrm{L}^{2} m^{2}}}, \quad . \quad . \quad . \quad . \quad . \quad .
$$

where $\Gamma$ signifies the maximum current. Or the current is the same as if the resistance were increased in the ratio of

$$
1: \sqrt{1+\left(\frac{\mathrm{L} m}{\mathrm{R}}\right)^{2}}
$$

If $\mathrm{L} m$ is large compared with $\mathrm{R}$, then $\Gamma$ is small compared with $\frac{\mathrm{E}}{\mathrm{R}}$, or the diminution of current-strength is large.

Let $\mathrm{T}$ be the time of a complete reversal. Then

$$
m=\frac{2 \pi}{\mathrm{T}} \text {. }
$$

With the Morse code, when $\mathrm{T}=\frac{1}{40}$ second, or $m=80 \pi$, an automatic transmitter produces 100 words per minute, or a little more. Let $\frac{L}{R}$ be between $\frac{1}{100}$ and $\frac{1}{10}$ second, then $\frac{L m}{R}$ is between $\frac{8 \pi}{10}$ and $8 \pi$, or (say) between 2.5 and 25 ; therefore the current will be reduced from $2 \cdot 5$ to 25 times.

On telephonic circuits, owing to the great rapidity of the reversals, the reduction in current-strength is great, and is nearly inversely proportional to the pitch of the tone, thus rendering it impossible to reproduce at the receiving end the same quality of sound as is emitted at the sending end, irrespective of mechanical or acoustical difficulties. The second partial tone of any continuous sound will be weakened twice as much as the first, the third thrice as much as the first, and so on, thus producing a general deadness or want of brilliancy.

It also appears from (2) that the resistance of the circuit becomes quite subordinate when $\frac{\mathrm{Lm}}{\mathbf{R}}$ is large; and it may then be greatly increased without much weakening the current. This is remarkably evident on telephonic circuits. On automatic circuits it has sometimes been found beneficial to introduce resistance-coils at the receiving end. The irregular effects of leakage from neighbouring wires, which mutilate 
the proper signals, are reduced in a greater proportion than the proper signals themselves.

3. Now let $R$ and $L$ belong to the electromagnet alone, and $R_{1}$ and $L_{1}$ be the resistance and electromagnetic capacity of the remainder of the circuit. Then (2) becomes

$$
\Gamma=\frac{E}{\sqrt{\left(\mathrm{R}+\mathrm{R}_{1}\right)^{2}+m^{2}\left(\mathrm{~L}+\mathrm{L}_{1}\right)^{2}}} . . .
$$

In solenoidal electromagnets, if $n$ is the number of windings of the wire in unit of length, and the number of layers in unit of thickness, $\mathrm{R}$ varies as $n^{4}$. $\mathrm{L}$ also varies as $n^{4}$, while the magnetizing force due to the unit current varies as $n^{2}$. Applying this to equation (3), if $\mathrm{F}$ is the magnetizing force, $F$ is a maximum, $n$ being variable, when

$$
\mathrm{R}^{2}+\mathrm{L}^{2} m^{2}=\mathrm{R}_{\mathrm{I}}^{2}+\mathrm{L}_{1}^{2} m^{2}, \text {. . . . . }
$$

$R_{1}$ and $L_{1}$ being considered constant, as belonging to the line. We may write (4) thus,

$$
\frac{\mathrm{R}}{\overline{\mathrm{R}}_{1}}=\sqrt{\frac{1+m^{2}\left(\frac{\mathrm{L}_{1}}{\mathrm{R}_{1}}\right)^{2}}{1+m^{2}\left(\frac{\mathrm{L}}{\mathrm{R}}\right)}} .
$$

Now $\frac{L_{1}}{\mathrm{R}_{1}}$ is constant for the same line-wire, whatever its length, since both $L_{1}$ and $R_{1}$ are proportional to the length of the line. Also $\frac{\mathrm{L}}{\mathrm{R}}$ is constant for the same coil, if only the diameter of the wire is variable, since both $\mathrm{L}$ and $\mathrm{R}$ vary as $n^{4}$. But the time interval $\frac{\mathrm{L}}{\mathrm{R}}$ for the electromagnet is in general much greater than the time interval $\frac{L_{1}}{R_{1}}$ for the line-wire; whence it follows, by inspection of (5), that $R$ must be much less than $R_{1}$ to produce the maximum magnetizing force; and the higher the speed, which is proportional to $m$, the less should $\mathrm{R}$ be.

The calculation of $\frac{\mathrm{I}_{1}}{\mathrm{R}_{1}}$ is easy, since the line-wire is long, straight, and parallel to the earth; but the calculation of $\frac{\tilde{L}}{\mathbf{R}}$ is not so easy, owing to the variety of shapes assumed by electromagnets used for telegraphic purposes, with their cores, polepieces, and armatures, which all influence the electromagnetic capacity, though they do not influence the resistance. It is therefore impossible to enunciate a general law, that the 
resistance of an electromagnet should be such or such a fraction of the external resistance ; for the result will be different, not only for every speed, but for every different construction of the electromagnet.

4. Approximate results are, however, easily obtainable in the case of a solenoidal electromagnet. Let its length be $l$, external radius $x$, internal radius $y$, with an iron core of radius $z$. Its electromagnetic capacity is

$$
\mathrm{L}=\frac{2}{3} \pi^{2} l n^{4}(x-y)^{2}\left(x^{2}+2 x y+3 y^{2}+24 \pi \kappa z^{2}\right) \quad . \quad .
$$

('Maxwell,' vol. ii. p. 283), where $\kappa$ is the coefficient of màgnetization of the core. Its resistance is

$$
\mathbf{R}=\pi \rho \ln ^{4}\left(x^{2}-y^{2}\right),
$$

where $\rho$ is the resistance of unit of length of wire of unit diameter. Therefore

$$
\overline{\mathrm{L}}=16 \pi^{2} \frac{\kappa}{\rho} \frac{x-y}{x+y} z^{2} . . . \quad . \quad .
$$

approximately, by leaving out $x^{2}+2 x y+3 y^{2}$ in (6) as small in comparison with $24 \pi \kappa z^{2}$, which is a large number, unless the core is very small. Let $\kappa=32$; also, if the specific resistance of copper be taken at $1.7 \mathrm{microhm}=1700 \mathrm{cs}$. s., then $\rho=1700 \times \frac{4}{\pi}$, and

$$
\frac{\mathrm{L}}{\mathrm{R}}=2 \cdot 33 \frac{x-y}{x+y} z^{2} \text { seconds. . . . . . }
$$

5. To determine $\frac{\mathrm{L}_{1}}{\mathrm{R}_{1}}$ for the line wire, Maxwell (vol. ii. p. 282) gives the coefficient of self-induction of a straight wire, the eircuit being completed by a parallel wire. The same method of calculation is applicable to any number of parallel straight wires, by finding the integral

$$
\mathrm{T}=\frac{1}{2} \iiint \mathrm{H} w d x d y d z
$$

where $\mathrm{T}$ is the kinetic energy of the system, and $\mathrm{H}, w$ are the vector-potential and the current at a point, both parallel to the axes of the wires. Thus, for $n$ parallel straight cylindrical wires of length $l$, conveying currents $\mathrm{C}_{1}, \mathrm{C}_{2}, \ldots$, of radii $a_{1}, a_{2}, \ldots$, specific magnetic capacities $\mu_{1}, \mu_{2}, \ldots$, representing the distance between the centre of two wires $m$ and $n$ by $b_{m n}$ we shall have

$$
\left.\begin{array}{rl}
\frac{2 \mathrm{~T}}{l} & =\frac{1}{2}\left(\mu_{1} \mathrm{C}_{1}^{2}+\mu_{2} \mathrm{C}_{2}^{2}+\ldots\right) \\
& -2 \mu_{0}\left(\mathrm{C}_{1}^{2} \log a_{1}+\mathrm{C}_{2}^{2} \log a_{2}+\ldots\right) \\
& -4 \mu_{0}\left(\mathrm{C}_{1} \mathrm{C}_{2} \log b_{12}+\mathrm{C}_{1} \mathrm{C}_{3} \log b_{13}+\mathrm{C}_{2} \mathrm{C}_{3} \log b_{23}+\ldots\right),
\end{array}\right\}
$$


with the sole condition

$$
\mathrm{C}_{1}+\mathrm{C}_{2}+\mathrm{C}_{3}+\ldots=0 \text {. }
$$

Let there be only four wires, 1 and 3 for one circuit, 2 and 4 for another; then $\mathrm{C}_{3}=-\mathrm{C}_{1}$, and $\mathrm{C}_{4}=-\mathrm{C}_{2}$. Substituting in $(10)$,

$$
\begin{aligned}
\frac{2 \mathrm{~T}}{l} & =\mathrm{C}_{1}^{2}\left(\frac{\mu_{1}+\mu_{3}}{2}+2 \mu_{0} \log \frac{b_{13}^{2}}{a_{1} a_{3}}\right) \\
& +\mathrm{C}_{2}^{2}\left(\frac{\mu_{2}+\mu_{4}}{2}+2 \mu_{0} \log \frac{b_{24}^{2}}{a_{2} a_{4}}\right) \\
& +2 \mathrm{C}_{1} \mathrm{C}_{2} \times 2 \mu_{0} \log \frac{b_{14} b_{23}}{b_{12} b_{34}} \text {. . . . . }
\end{aligned}
$$

The coefficient of $\mathrm{C}_{\mathrm{w}}^{2}$ in (11) is the coefficient of self-induction per unit of length of the circuit conveying the current $\mathrm{C}_{1}$. Similarly for $\mathrm{C}_{2}^{2}$; and the coefficient of $2 \mathrm{C}_{1} \mathrm{C}_{2}$ is the coefficient of mutual induction per unit of length of the two circuits.

From (10) we may find the coefficients of induction of suspended wires, the circuit being completed through the earth. Let $M$ be the coefficient of mutual induction, and $\mathrm{L}_{1}, \mathrm{~L}_{2}$, the coefficients of self-induction of two wires of radii $a_{1}, a_{2}$, heights above ground $h_{1}, h_{2}$, horizontal distance apart $d$, and specific magnetic capacities $\mu_{1}, \mu_{2}$; then

$$
\left.\begin{array}{l}
\frac{\mathrm{L}_{1}}{l}=\frac{\mu_{1}}{2}+2 \log \frac{2 h_{1}}{a_{1}}, \\
\frac{\mathrm{L}_{2}}{l}=\frac{\mu_{2}}{2}+2 \log \frac{2 h_{2}}{a_{2}}, \\
\frac{\mathrm{M}}{l}=\log \frac{d^{2}+\left(h_{1}+h_{2}\right)^{2}}{d^{2}+\left(h_{1}-h_{2}\right)^{2}},
\end{array}\right\}, \quad . \quad .
$$

where $\mu_{0}$ is made equal to unity.

As a practical case, let

$$
h_{1}=h_{2}=3 \text { metres, }
$$

$a_{1}=a_{2}=\cdot 002$ metre, $\mu_{1}=\mu_{2}=1+4 \pi \kappa=315$, if $\kappa=25$, and $d=\cdot 5$ metre. Then

$$
\begin{aligned}
\mathrm{L}_{1}=\mathrm{L}_{2} & =173, \\
\mathrm{M} & =5
\end{aligned}
$$

approximately. Also, if the resistance is 13 ohms per mile, the resistance per centimetre is $80778 \mathrm{c.}$ g. s. ; therefore

$$
\frac{\mathrm{L}_{1}}{\mathbf{R}_{1}}=\frac{173}{80778}=\cdot 00214 \text { second. . . . . }
$$

6. This time interval being in general very small compared 
with $\frac{L}{R}$ for the electromagnet, we may neglect it; and then (5) becomes

$$
\frac{\mathrm{R}}{\mathrm{R}_{1}}=\frac{1}{\sqrt{1+m^{2} \frac{\mathrm{L}^{2}}{\mathrm{R}^{2}}}},
$$

or $\mathrm{R}_{1}=\mathrm{L} m$ approximately. The resistance of the electromagnet for high speeds varies inversely as the speed to obtain the maximum strength of signals.

Using the value of $\frac{L}{R}$ given in (9), we have

$$
\frac{\mathrm{R}}{\mathbf{R}_{1}}=\frac{\mathrm{T}}{14 \cdot 64 \frac{x-y}{x+y} \cdot z^{2}} .
$$

where $\mathrm{T}=\frac{2 \pi}{m}$.

At 100 words per minute Morse code, $T=$ about $\frac{1}{40}$ second; therefore at this speed

$$
\frac{\mathrm{R}_{1}}{\mathbf{R}}=585 \cdot 6 \frac{x-y}{x+y} z^{2} .
$$

Suppose $x=2, y=z=1$ centimetre; then

$$
\frac{R_{1}}{\mathrm{R}}=195 \cdot 2 ;
$$

or the resistance of the electromagnet is $\frac{1}{195}$ th part of the external resistance to obtain the maximum magnetizing forcea very low result. If $\frac{L_{1}}{R_{1}}$ is taken into account, this becomes $T^{\frac{1}{7}}$ th.

7. Having made the magnetizing force a maximum for a given speed and size of electromagnet, by varying the thickness of the wire, we may next find the ratio between the outer and inner radius of the coil to make the attractive force between the core and a soft-iron armature a maximum. Wo have

(neglecting $\mathrm{L}_{1}$ ), where

$$
\Gamma=\frac{\mathrm{E}}{\sqrt{\left(\mathbf{R}+\mathrm{R}_{1}\right)^{2}+\mathrm{L}^{2} m^{2}}}
$$

$$
\begin{aligned}
& \mathrm{L}=\frac{2}{3} \pi^{2} l n^{4}(x-y)^{2}\left(x^{2}+2 x y+3 y^{2}+24 \pi \kappa z^{2}\right), \\
& \mathrm{R}=\pi \rho \ln \left(x^{4}-y^{2}\right) .
\end{aligned}
$$


Also

$$
\mathrm{F}=\Gamma \mathrm{G},
$$

where $\mathrm{F}$ is the magnetizing force, and

$$
\mathrm{G}=4 \pi n^{2}(x-y) \text {. }
$$

To make $\mathrm{F}$ a maximum, $n$ being variable, we found

Therefore

$$
\mathrm{R}^{2}+\mathrm{L}^{2} m^{2}=\mathrm{R}_{\mathrm{l}}^{2} \text {. }
$$

Substituting

$$
F=\frac{E G}{R_{1} \sqrt{2\left(1+\frac{\bar{R}}{R_{1}}\right)}} .
$$

we have

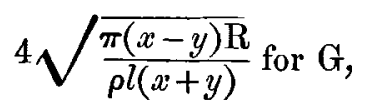

$$
\mathbf{F}^{2}=\frac{\frac{8 \pi(x-y) \mathrm{E}^{2}}{\rho l(x+y)}}{\mathbf{R}_{1}\left(1+\frac{\mathbf{R}_{1}}{\mathrm{R}}\right)}
$$

Now

$$
\begin{aligned}
\frac{\mathrm{R}_{1}}{\mathrm{R}} & =\sqrt{1+\frac{\mathrm{L}^{2} m^{2}}{\mathrm{R}^{2}}}=\frac{\mathrm{L} m}{\mathrm{R}} \text { approximately, } \\
& =\frac{2 m \pi}{3 \rho} \frac{x-y}{x+y}\left(x^{2}+2 x y+3 y^{2}+24 \pi \kappa z^{2}\right) .
\end{aligned}
$$

Therefore

$$
\mathrm{F}^{2}=\frac{\frac{12 \mathrm{E}^{2}}{\mathrm{R}_{1}}}{\operatorname{lm}\left(x^{2}+2 x y+3 y^{2}+24 \pi \kappa z^{2}\right)+\frac{3 \rho l}{2 \pi} \frac{x+y}{x-y}} .
$$

Now the magnetization of the core is proportional to the magnetizing force; and the attractive force between the core and a soft-iron armature placed close to it is proportional to the square of the magnetization and to the cross section of the core. Therefore, if $\mathrm{A}$ is the attractive force,

$$
\mathrm{A} \propto \frac{z^{2}}{\ln \left(x^{2}+2 x y+3 y^{2}+24 \pi \kappa z^{2}\right)+\frac{3 \rho l}{2 \pi} \frac{x+y}{x-y}} .
$$


This increases with $z$; therefore let $z=y$, the inner radius of the coil ; let $x$ be constant and $y$ variable ; then $A$ is a maximum when

$$
\frac{x^{2}}{y^{2}}+\frac{2 x}{y}+\frac{3 \rho}{2 \pi y^{2} m} \frac{x+y}{x-y}
$$

is a minimum; and that is when

$$
\frac{2 m \pi}{3 \rho} x^{2}=\frac{\frac{y}{x}-\left(1-\frac{y^{2}}{x^{2}}\right)}{\left(1-\frac{y}{x}\right)\left(1-\frac{y^{2}}{x^{2}}\right)}
$$

The least value of $\frac{y}{x}$ is

$$
\frac{y}{x}=\frac{\sqrt{ } 5-1}{2}=\cdot 618 \text {. }
$$

Using the former value of $\rho$, viz. $1700 \times \frac{4}{\pi}$, also $m=80 \pi$ and $x=2$ centimetres, then

$$
\frac{y}{x}=\cdot 7 \text { nearly. }
$$

$\frac{y}{x}$ increases very slowly as $x$ and $m$ increase.

In this determination of $\frac{y}{x}$, the outer radius has been supposed to be constant, and the inner variable, and with it the iron core. If, on the other hand, the inner radius is fixed and the outer variable, a different ratio is obtained, viz.

$$
\frac{2 m \pi}{3 \rho} x^{2}=\frac{\frac{y}{x}}{\left(1-\frac{y}{x}\right)\left(1-\frac{y^{2}}{x^{2}}\right)},
$$

which gives lower valnes to $\frac{y}{x}$ than before.

It also appears from (14) that the attractive force varies inversely as the length of the coil. Though the formulæ are only true for long coils, yet it points in the direction of short, flat coils; for the attractive force is also increased by increasing the transverse dimensions of the coil.

8. In paragraph 5 we found $L_{1}=173$ and $M=5$ approximately for iron wires of a certain size, distance apart, and 
height above ground. The ratio

$$
\frac{\mathrm{L}_{1}}{\mathrm{M}}=34
$$

expresses the ratio of the strength of the sinal wave currents in the wire containing the electromotive force $\mathrm{E} \sin m t$ to the corresponding induced currents in the parallel wire, or, rather, the minimum value of that ratio for rapid reversals. For if $R_{1}, L_{1}$ belong to the primary circuit, $R_{2}, L_{2}$ to the secondary, then

$$
\Gamma_{1}=\frac{\mathrm{E} \sqrt{\mathrm{R}_{2}^{2}+\mathrm{L}_{2}^{2} m^{2}}}{\sqrt{\left\{\mathrm{R}_{1} \mathrm{R}_{2}-m^{2}\left(\overline{\mathrm{L}}_{1} \overline{\mathrm{L}}_{2}-\mathrm{M}^{2}\right)\right\}^{2}+m^{2}\left(\overline{\mathrm{R}}_{1} \mathrm{~L}_{2}+\mathrm{R}_{2} \overline{\mathrm{L}}_{1}\right)^{2}}},
$$

and

$$
\frac{\Gamma_{2}}{\Gamma_{1}}=\frac{\mathrm{M} m}{\sqrt{\mathrm{R}_{2}^{2}+\mathrm{L}_{2}^{2} m^{2}}}
$$

As $m$ increases, this approximates to

$$
\frac{\Gamma_{2}}{\Gamma_{1}}=\frac{M}{\mathrm{~L}_{2}}
$$

equal to about $\frac{1}{34}$ if there are no electromagnets in the second. ary circuit, otherwise much less. It is here assumed that $\kappa=25$ for the iron wire.

XXV. On the Effect of Variation of Pressure on the Length of Disruptive Discharge in Air. By J. E. H. Gondon, B.A., Assistant General Secretary of the British Association*.

\section{[Plate III.]}

\section{History.}

TN 1834 Mr. Snow Harris stated $\dagger$ that, other things being I equal, the length of the spark which an electric machine or Leyden jar will give in air varies in the simple inverse ratio of the pressure. He, however, gives no tables or figures in support of his law.

Sir William Thomson $\ddagger$ has determined, by means of an absolute electrometer, the difference of potentials corresponding

* Communicated by the Author, having been read before Section A of the British Association, Dublin, 1878.

+ Philosophical Transactions.

t Proc. Roy. Soc. 1860, Papers on Electrostatics, p. 247. 